\title{
Physical, Psychological and Social Frailtyin Prediction of Disability and Quality of Life
}

T. Coelho, C. Paúl, L. Fernandes

Introduction: Frailty is a state of increased vulnerability that entails a high risk of adverse outcomes. While traditional approaches define frailty as an exclusively physical condition, there is an increasing number of authors that include psychological and social components in the conceptualization of the syndrome.

Objective: Assuming a multidimensional approach to frailty, this study aims to examine which domain (physical, psychological or social) is the most significant predictor of disability and quality of life.

Methods: A longitudinal study was designed recruiting 95 community-dwelling elderly. Frailty domains were assessed at baseline with the Tilburg Frailty Indicator. Disability in basic and instrumental activities of daily living/ADL and IADL were measured with the Barthel Index and with the Lawton and Brody Scale. Quality of life was evaluated with EUROHIS-QOL-8 and WHOQOL-OLD.

Results: The mean age of the participants was $78.5 \pm 6.2$ years, and most were women (67.4\%). After controlling for life-course determinants and comorbidity, physical frailty contributed to the prediction of most of the adverse outcomes: ADL disability (3.3\%), IADL disability (2.2\%), global quality of life (EUROHIS-QOL8: $4.7 \%$; WHOQOL-OLD: $2.9 \%$ ) and quality of life facets: sensory abilities (4.5\%), social participation $(5.6 \%)$, death and dying (3.0\%) and family/family life (2.8\%). Psychological frailty predicted past, present and future activities $(7.2 \%)$ and intimacy $(4.0 \%)$, whereas the effect of social frailty was not significant when compared with the other domains.

Conclusions: The results of the present study highlight the relevance of physical factors, but also the importance at least of the psychological components, in the definition of frailty. 